\title{
Evaluation of sample size effect on the identification of haplotype blocks
}

\author{
Dai Osabe 1,2, Toshihito Tanahashi*3, Kyoko Nomura1,2, Shuichi Shinohara4, \\ Naoto Nakamura ${ }^{5}$, Toshikazu Yoshikawa ${ }^{5}$, Hiroshi Shiota ${ }^{6}$, \\ Parvaneh Keshavarz ${ }^{3}$, Yuka Yamaguchi3 ${ }^{3}$, Kiyoshi Kunika3 ${ }^{3}$, Maki Moritani ${ }^{3}$, \\ Hiroshi Inoue ${ }^{3}$ and Mitsuo Itakura ${ }^{2,3}$
}

\begin{abstract}
Address: ${ }^{1}$ Department of Bioinformatics, Division of Life Science Systems, Fujitsu Limited, 1-5-2, Higashishinbashi, Minato-ku, 105-7123, Tokyo, Japan, ${ }^{2}$ Section for Diabetes, Genotyping Division, Genetic Diversification Analysis Project, Japan Biological Information Consortium (JBIC), Tokyo, Japan, ${ }^{3}$ Division of Genetic Information, Institute for Genome Research, the University of Tokushima, 3-18-15, Kuramoto-cho, 770-8503, Tokushima, Japan, ${ }^{4}$ Division of R\&D Solution, Fujitsu Nagano Systems Engineering Limited, 380-3813, Nagano, Japan, ${ }^{5}$ Department of Endocrinology and Metabolism, Kyoto Prefectural University of Medicine Graduate School of Medical Sciences, 465, Kajii-cho, HirokojiKawaramachi, Kamigyo-ku, Kyoto, 602-8566, Japan and ${ }^{6}$ Department of Ophthalmology and Visual Neuroscience, Institute for Health Biosciences, the University of Tokushima, 3-18-15, Kuramoto-cho, Tokushima, 770-8503, Japan

Email: Dai Osabe -d_osabe@jp.fujitsu.com; Toshihito Tanahashi* - tana@genome.tokushima-u.ac.jp; Kyoko Nomura - nomura.kyoko@jp.fujitsu.com; Shuichi Shinohara - shino@nagano.fujitsu.com; Naoto Nakamura - naoto@koto.kpu-m.ac.jp; Toshikazu Yoshikawa - toshi@koto.kpu-m.ac.jp; Hiroshi Shiota - shiota@clin.med.tokushima-u.ac.jp; Parvaneh Keshavarz - parvaneh@genome.tokushima-u.ac.jp; Yuka Yamaguchi - yama@genome.tokushima-u.ac.jp; Kiyoshi Kunika - kunika@genome.tokushima-u.ac.jp; Maki Moritani - moritani@genome.tokushima-u.ac.jp; Hiroshi Inoue - hinoue@genome.tokushima-u.ac.jp; Mitsuo Itakura - itakura@genome.tokushima-u.ac.jp

* Corresponding author
\end{abstract}

Published: 14 June 2007

BMC Bioinformatics 2007, 8:200

doi:10.1|86/|47|-2105-8-200
Received: 28 December 2006

Accepted: 14 June 2007

This article is available from: http://www.biomedcentral.com/I47I-2105/8/200

(C) 2007 Osabe et al; licensee BioMed Central Ltd.

This is an Open Access article distributed under the terms of the Creative Commons Attribution License (http://creativecommons.org/licenses/by/2.0), which permits unrestricted use, distribution, and reproduction in any medium, provided the original work is properly cited.

\begin{abstract}
Background: Genome-wide maps of linkage disequilibrium (LD) and haplotypes have been created for different populations. Substantial sharing of the boundaries and haplotypes among populations was observed, but haplotype variations have also been reported across populations. Conflicting observations on the extent and distribution of haplotypes require careful examination. The mechanisms that shape haplotypes have not been fully explored, although the effect of sample size has been implicated. We present a close examination of the effect of sample size on haplotype blocks using an original computational simulation.

Results: A region spanning 19.3I Mb on chromosome 20q was genotyped for I, I 47 SNPs in 725 Japanese subjects. One region of $445 \mathrm{~kb}$ exhibiting a single strong $L D$ value (average $\left|\mathrm{D}^{\prime}\right| ; 0.94$ ) was selected for the analysis of sample size effect on haplotype structure. Three different block definitions (recombination-based, LD-based, and diversity-based) were exploited to create simulations for block identification with $\theta$ value from real genotyping data. As a result, it was quite difficult to estimate a haplotype block for data with less than 200 samples. Attainment of a reliable haplotype structure with 50 samples was not possible, although the simulation was repeated 10,000 times.

Conclusion: These analyses underscored the difficulties of estimating haplotype blocks. To acquire a reliable result, it would be necessary to increase sample size more than 725 and to repeat the simulation 3,000 times. Even in one genomic region showing a high LD value, the haplotype block might be fragile. We emphasize the importance of applying careful confidence measures when using the estimated haplotype structure in biomedical research.
\end{abstract}




\section{Background}

There is a great interest in using genetic association studies to identify the disease-susceptibility variants related to the common complex diseases. To design these studies appropriately, it is important to understand the feature of linkage disequilibrium (LD) in candidate genes or genomic regions of interest [1]. Several studies have shown that the human genome contains regions of high LD value with low haplotype diversity by a small number of SNPs [2-4]. These regions are called haplotype block, each of which reflects the descent from a single ancient ancestral chromosome. The construction of a haplotype block is one way to reduce the complexity of the problem of association mapping of the common complex diseases.

Haplotype blocks are defined computationally by various algorithms. In general, they are classified into the following categories: recombination-based, LD-based, and diversity-based methods. These block definitions are consistent with a block-covered sequence, which is considered a block as a part of the genomic sequence. However, the haplotype block border is not usually stable, and blocks can fall into sub-blocks within the border [5].

The general properties of haplotype block construction in the human genome are not well understood. Thus, the International Haplotype Map (HapMap) project has genotyped a huge number of SNPs in samples from subjects of Caucasian, African, and Asian descent to better understand the human haplotype structure [6]. Several studies (including HapMap) have shown differences in LD and haplotype block patterns in populations and chromosomes. In addition, it was revealed that SNPs ascertainment, selection and spacing could explain the observed block length $[5,7]$, and that SNPs density has a crucial influence on the length of method-defined blocks [8]. Despite the extensive empirical studies on haplotype blocks, there is no definitive answer as to how sample size impacts the assessment of block structure. For example, a study on chromosome 21 examined 20 independent subjects from diverse populations [2]. Even for a relatively large data set, it contained only 275 individual samples, leading to 400 independent chromosomes [4]. Thus, it was possible that the detected block structure was dependent on the small number of samples, and this seemed a preliminary finding. It remains unclear how many individuals are needed to acquire reliable features of haplotype block [5,9].

In this study, we developed a simulation with random resampling from real genotyping data in 725 Japanese and introduced an original measurement of $\theta$ value for the identification of haplotype block defined by three algorithms. With the original measurement, we focused on haplotype block structure, especially within a high LD region. We further assessed the robustness of haplotype blocks estimated under the different sample size conditions.

\section{Results}

\section{Selection of the analyzed region}

One region was selected on chromosome 20q11.22, in which a single strong $\left|D^{\prime}\right|$ block was observed without substantial recombination in the current population (Fig. 1A). However, the region was broken into small blocks by $\mathrm{r}^{2}$. The average values of $\left|\mathrm{D}^{\prime}\right|$ and $\mathrm{r}^{2}$ were 0.94 and 0.59 , respectively. Based on NCBI human build 35, the total length was about $445 \mathrm{~kb}$ from $32,311,428$ to $32,756,554$ bp. This region was composed of 37 SNPs, and the average distance between SNPs was $12.4 \mathrm{~kb}$. SNPs with MAF greater than 0.1 (average MAF 0.36) were used for subsequent study. Detailed information on selected SNPs is shown in Table 1.

\section{Use of a novel measurement: $\theta$ value}

An original measurement ( $\theta$ value) was used for block identification. The $\theta$ value represents the probability of whether a SNPs interval resides within or outside a haplotype block. The word "interval" as used here refers to the region between two adjacent SNPs (See Methods). Using the $\theta$ value with more than two block definitions allows an estimation of the suitable structure of the haplotype block in the region of interest.

In the analyzed region, different reference haplotype blocks were identified with three separate algorithms, despite a single strong $\left|\mathrm{D}^{\prime}\right|$ value (Fig. $1 \mathrm{~B}$ ). To evaluate the discrepancy in block identification, $\theta$ profiles were calculated for all pairwise SNPs with a flow chart in Fig. 2.

The influence of the mixture of cases with control samples was examined first. $\theta$ profiles calculated with only controls ( $x=358$ and $N=3,000)$ were compared to the mixed data of controls and cases $(x=358$ and $N=3,000)$. The mean square errors (MSE) between the two data sets was calculated, where MSE was the sum of the variance and squared bias of the estimates [10]. The observed MSE values were under $0.005(0.5 \%)$ ranged from 0.00384 to 0.00305 (Additional file 1). Similarly, when $\theta$ profiles were compared with mixed data from 725 samples $(N=$ 3,000 ), the MSE values were under 0.005 , leading to a reasonable accuracy for the combination of the two groups (Additional file 1). This calculation suggested that the profound bias of the mixture of cases was rather low. In addition, there were no significant differences (Chisquare $\mathrm{P}<0.01$ ) in allele frequencies with all 37 SNPs in a case-control association study and all SNPs satisfied with HWE test $(\mathrm{P}<0.05)$. As a result, the genotyping data from 367 cases and 358 controls were merged for a total of 725 samples of Japanese genotyping data. 


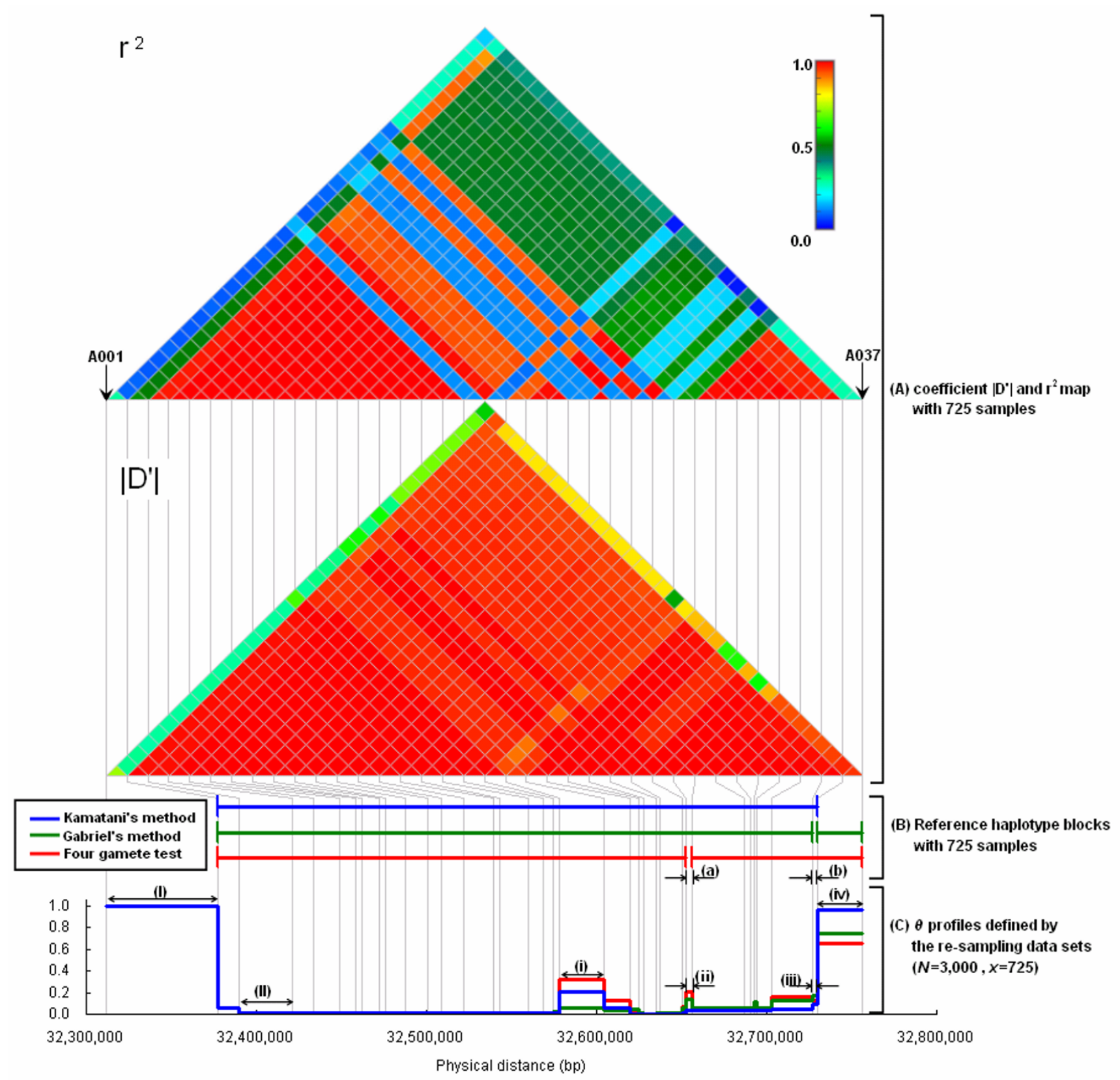

\section{Figure I}

(A) Map of linkage disequilibrium (LD) coefficient |D'| (lower part) and $r^{2}$ (upper part) within the analyzed region on chromosome $20 \mathrm{q}$ I I.22 (445 kb). A single strong |D'| block (average 0.94) is observed, but broken into small blocks with $\mathrm{r}^{2}$. (B) Structure of reference haplotype blocks with three different algorithms (Kamatani's method, Gabriel's method, and four-gamete test) using 725 samples. Each haplotype block is shown as blue (Kamatani's), green (Gabriel's), or red (four-gamete test) horizontal bars. Two gaps of (a) and (b) were observed in the analyzed region ((a) in the four-gamete test, and (b) in Gabriel's method). (C) $\theta$ profiles with the maximum number of samples $(x=725)$, and 3,000 repeats $(N=3,000)$. $\theta$ profiles show values between 0 and $\mathrm{I}$ based on its definition. The scale of $\theta$ profiles is shown on the vertical axis. The physical distance on chromosome $20 \mathrm{q}$ is shown on the horizontal axis. SNPs positions are presented as vertical gray bar through $(\mathrm{A})$ to $(\mathrm{C})$. Based on the complete identification of $\theta$ profiles, two SNPs intervals of (I) and (II) were selected for further analysis. We also selected four SNPs intervals from (i) to (iv), showing the variations of $\theta$ profiles, for further analysis. (A), (B), and (C) are illustrated at the same physical scale. 
Table I: List and information of 37 SNPs in the analyzed region on chromosome 20q I I.22

\begin{tabular}{|c|c|c|c|c|c|c|c|c|c|c|c|c|c|}
\hline \multirow[t]{2}{*}{ No. } & \multirow[t]{2}{*}{ dbSNP ID } & \multirow[t]{2}{*}{ Position } & \multicolumn{2}{|c|}{ No. of used samples } & \multicolumn{3}{|c|}{ Minor allele frequency } & \multicolumn{3}{|c|}{$P$ value (HWE test) } & \multicolumn{2}{|c|}{$P$ value (association test) } & \multirow[t]{2}{*}{ Odds ratio } \\
\hline & & & Control & Case & Control & Case & Overall & Control & Case & Overall & Allele & Genotype & \\
\hline A00I & rs819135 & $32,311,428$ & 357 & 362 & 0.40 & 0.34 & 0.37 & 0.32 & 0.30 & 0.18 & 0.03 & 0.08 & 1.27 \\
\hline A002 & rs6088466 & $32,377,195$ & 358 & 361 & 0.46 & 0.50 & 0.48 & 0.84 & 0.79 & 0.99 & 0.17 & 0.37 & 1.16 \\
\hline A003 & rsI205336 & $32,389,997$ & 358 & 367 & 0.36 & 0.35 & 0.36 & 0.47 & 0.81 & 0.50 & 0.85 & 0.93 & 1.02 \\
\hline A004 & rs3746455 & $32,420,877$ & 357 & 367 & 0.36 & 0.35 & 0.35 & 0.72 & 0.87 & 0.72 & 0.86 & 0.98 & 1.02 \\
\hline A005 & rs6058029 & $32,433,304$ & 352 & 367 & 0.35 & 0.35 & 0.35 & 0.44 & 0.87 & 0.51 & 0.94 & 0.90 & 1.01 \\
\hline A006 & rs6087579 & $32,448,816$ & 354 & 367 & 0.36 & 0.35 & 0.36 & 0.78 & 0.87 & 0.76 & 0.86 & 0.98 & 1.02 \\
\hline A007 & rs6579165 & $32,458,376$ & 357 & 359 & 0.36 & 0.36 & 0.36 & 0.72 & 0.71 & 0.61 & 0.98 & 1.00 & 1.00 \\
\hline A008 & rs $49 \mid 1420$ & $32,462,315$ & 358 & 365 & 0.36 & 0.35 & 0.35 & 0.80 & 0.84 & 0.75 & 0.74 & 0.95 & 1.04 \\
\hline A009 & rs4277599 & $32,472,566$ & 356 & 360 & 0.36 & 0.35 & 0.35 & 0.70 & 0.74 & 0.61 & 0.79 & 0.96 & 1.03 \\
\hline A0I0 & rs2424992 & $32,475,721$ & 354 & 366 & 0.36 & 0.35 & 0.35 & 0.67 & 0.80 & 0.63 & 0.85 & 0.97 & 1.02 \\
\hline A0II & rs6120644 & $32,487,47 \mid$ & 355 & 364 & 0.36 & 0.36 & 0.36 & 0.92 & 0.81 & 0.81 & 0.94 & 0.99 & 1.01 \\
\hline $\mathrm{A} 012$ & rs3736762 & $32,500,997$ & 357 & 361 & 0.36 & 0.36 & 0.36 & 0.72 & 0.87 & 0.71 & 0.96 & 0.99 & 1.01 \\
\hline $\mathrm{A} 013$ & rs6059850 & $32,508,445$ & 352 & 364 & 0.36 & 0.35 & 0.35 & 0.98 & 0.70 & 0.77 & 0.76 & 0.92 & 1.03 \\
\hline $\mathrm{A} 014$ & rs6059856 & $32,521,615$ & 355 & 367 & 0.36 & 0.35 & 0.36 & 0.92 & 0.87 & 0.85 & 0.85 & 0.98 & 1.02 \\
\hline A0I5 & rs6059866 & $32,539,47 \mid$ & 355 & 360 & 0.36 & 0.35 & 0.35 & 0.92 & 0.92 & 0.89 & 0.80 & 0.97 & 1.03 \\
\hline A016 & rs6059868 & $32,543,|2|$ & 357 & 366 & 0.36 & 0.35 & 0.35 & 0.72 & 0.91 & 0.75 & 0.85 & 0.97 & 1.02 \\
\hline A017 & rs60885I2 & $32,559,552$ & 355 & 365 & 0.36 & 0.35 & 0.36 & 0.86 & 0.83 & 0.78 & 0.86 & 0.98 & 1.02 \\
\hline $\mathrm{A} 018$ & rs6120669 & $32,568,689$ & 356 & 367 & 0.36 & 0.35 & 0.36 & 0.88 & 0.87 & 0.83 & 0.83 & 0.98 & 1.02 \\
\hline A019 & rsII22174 & $32,574,507$ & 356 & 367 & 0.18 & 0.15 & 0.16 & 0.41 & 0.97 & 0.51 & 0.09 & 0.22 & 1.27 \\
\hline A020 & rs6087592 & $32,578,164$ & 353 & 367 & 0.35 & 0.36 & 0.35 & 0.66 & 0.96 & 0.79 & 0.87 & 0.92 & 1.02 \\
\hline A02I & rsIII67239 & $32,604,133$ & 354 & 363 & 0.36 & 0.36 & 0.36 & 0.88 & 0.72 & 0.72 & 0.85 & 0.97 & 1.02 \\
\hline A022 & rs6088527 & $32,619,502$ & 354 & 361 & 0.36 & 0.35 & 0.36 & 0.90 & 0.48 & 0.56 & 0.83 & 0.90 & 1.02 \\
\hline A023 & rs764597 & $32,624,886$ & 357 & 365 & 0.36 & 0.36 & 0.36 & 0.89 & 0.65 & 0.67 & 0.92 & 0.97 & 1.01 \\
\hline A024 & rs2889849 & $32,627,938$ & 352 & 365 & 0.18 & 0.15 & 0.16 & 0.32 & 0.41 & 0.80 & 0.11 & 0.13 & 1.26 \\
\hline A025 & rs932542 & $32,635,029$ & 357 & 367 & 0.18 & 0.15 & 0.16 & 0.45 & 0.42 & 0.94 & 0.15 & 0.19 & 1.23 \\
\hline A026 & rs2295444 & $32,637,544$ & 354 & 360 & 0.36 & 0.35 & 0.36 & 0.93 & 0.47 & 0.65 & 0.73 & 0.80 & 1.04 \\
\hline A027 & rs2378199 & $32,650,|4|$ & 358 & 360 & 0.17 & 0.14 & 0.16 & 0.26 & 0.36 & 0.73 & 0.07 & 0.08 & 1.30 \\
\hline A028 & rs6088536 & $32,652,767$ & 358 & 359 & 0.36 & 0.36 & 0.36 & 0.97 & 0.65 & 0.77 & 0.88 & 0.93 & 1.02 \\
\hline A029 & rs6|4|488 & $32,656,407$ & 356 & 367 & 0.46 & 0.49 & 0.48 & 0.24 & 0.71 & 0.56 & 0.24 & 0.28 & 1.13 \\
\hline A030 & rs6142210 & $32,686,673$ & 355 & 361 & 0.46 & 0.49 & 0.48 & 0.26 & 0.56 & 0.70 & 0.28 & 0.27 & 1.12 \\
\hline A03I & rs6088552 & $32,690,152$ & 358 & 366 & 0.46 & 0.49 & 0.48 & 0.24 & 0.60 & 0.64 & 0.22 & 0.23 & 1.14 \\
\hline A032 & rs7269596 & $32,692,724$ & 352 & 365 & 0.47 & 0.50 & 0.48 & 0.27 & 0.64 & 0.65 & 0.26 & 0.29 & 1.13 \\
\hline A033 & rs 6087612 & $32,694,483$ & 352 & 367 & 0.46 & 0.50 & 0.48 & 0.29 & 0.64 & 0.67 & 0.16 & 0.21 & 1.16 \\
\hline A034 & rs 4911158 & $32,703,173$ & 357 & 363 & 0.46 & 0.50 & 0.48 & 0.31 & 0.56 & 0.74 & 0.18 & 0.22 & 1.15 \\
\hline A035 & rs6087616 & $32,726,694$ & 356 & 364 & 0.46 & 0.50 & 0.48 & 0.46 & 0.60 & 0.86 & 0.18 & 0.28 & 1.15 \\
\hline A036 & rs1321306 & $32,730,040$ & 353 & 365 & 0.46 & 0.49 & 0.48 & 0.22 & 0.71 & 0.54 & 0.23 & 0.26 & 1.13 \\
\hline A037 & rs910870 & $32,756,554$ & 355 & 360 & 0.25 & 0.23 & 0.24 & 0.56 & 0.64 & 0.93 & 0.31 & 0.45 & 1.13 \\
\hline
\end{tabular}

Genome position is based on the NCBI build 35. The number of called sample is the number after excluding the undetermined sample(s) with TaqMan assay. HWE denotes Hardy-Weinberg equilibrium. $95 \% \mathrm{Cl}$ denotes $95 \%$ confidence interval. The crude odds ratio was calculated for the allele model. 


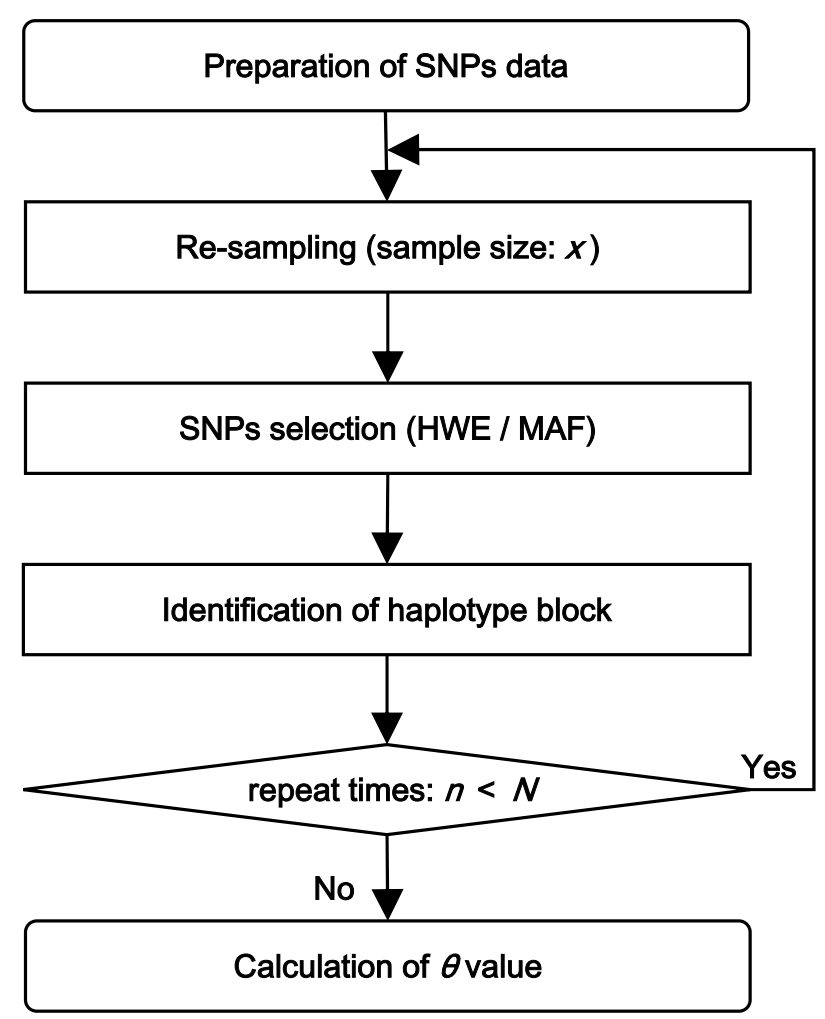

Figure 2

A flow chart of computational simulation. This flow chart outlines the basic algorithms including re-sampling, haplotype block definition, and block concordance (See Methods). $x$ is the sample size in the re-sampling process. This parameter is increased from 50 to 700 in increments of 50 , in addition to 10,25 , and $725 . n$ is the number of repeat times of simulation under the maximum repeat time $N$. The $\theta$ value is an original measurement of the estimation of haplotype block, and is the ratio of times to $N$ times when simulating the transition zone of block (See Materials and Methods). HWE denotes HardyWeinberg equilibrium, and MAF denotes minor allele frequencies.

With the maximum sample size of 725 subjects and 3,000 repeat times, $\theta$ profiles were plotted against the physical position of the SNPs (Fig. 1C). The complete concordance of haplotype blocks was observed in some intervals (e.g., (I) and (II)), but not in others (e.g., (i), (ii), (iii), and (iv)). It may be difficult to clearly identify the haplotype block even for the $\theta$ value. For subsequent analyses, two intervals, (I) and (II), were selected as the complete concordance regions of the block, and four intervals from (i) to (iv) were defined as incomplete concordance regions.

\section{Reference haplotype blocks and $\theta$ value}

Although there was a difference among reference haplotype blocks across the analyzed region (Fig. 1B), $\theta$ profiles generated by the three algorithms were comparatively similar (Fig. 1C). We compared $\theta$ profiles with reference haplotype blocks defined by 725 samples.

In the interval (a), a reference haplotype block was disrupted by the four-gamete test, but it was continuous with the other two methods (Fig. 1B). This finding, supported by the intermediate $\mathrm{r}^{2}$ value, identified interval (a) as the fragile region of haplotype block. In contrast, $\theta$ profiles showed low values (less than 0.2 ) with all three algorithms in the corresponding interval (interval (ii) in Fig. 1C). Namely, interval (a) was identified as the transition zone in $20 \%$ of 3,000 simulations of the $\theta$ values. Interpretation of the $\theta$ profiles reveals that interval (a) might be included in the transition zone of the block, and that LD might be disrupted against the estimation of Gabriel's and Kamatani's methods.

All reference haplotype blocks were continuous in the interval (i) (Fig. 1B). However, four-gamete test showed a $\theta$ value of 0.30 after 3,000 simulations and a divided interval (Fig. 1C). In addition, the $\theta$ value was not zero with the other two algorithms. Similar discrepancies were observed between the $\theta$ value and reference blocks defined by a single algorithm with two other intervals (iii) and (iv). It was quite difficult to clearly identify the block structure in these SNPs intervals. These results suggest that haplotype blocks might be fragile with one block algorithm, even if the region showed a single strong $L D$ value defined by $\left|D^{\prime}\right|$.

\section{Data simulation by $\theta$ value}

To evaluate the effect of sample size on the identification of haplotype blocks, $\theta$ profiles were calculated with a variable number of sample sizes $(x)$. Fig. 3 left side panels show the results obtained with intervals (I) and (II). Within these two intervals, complete concordance was observed between the $\theta$ value and reference block definitions. The $\theta$ value could increase or decrease to the maximum or minimum value ( 1 or 0 ) dependent on the sample size, and it converged when using more than 200 samples. This result suggests that sample size could have an effect on the identification of haplotype block, and it was not possible to obtain a reliable block identification for data with less than 200 samples.

There was also a difficulty with haplotype block definitions within the other four intervals ((i), (ii), (iii), and (iv)) in spite of the estimation by the $\theta$ value. The $\theta$ values in these intervals were more strongly influenced by sample size than those in intervals (I) and (II). In particular, the $\theta$ value did not converge even for calculations with more than 600 samples. This might imply that the precise identification of haplotype block was difficult for data with 725 samples. However, the exact number of samples 


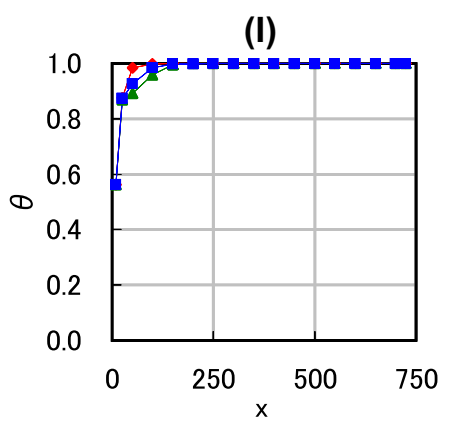

(II)

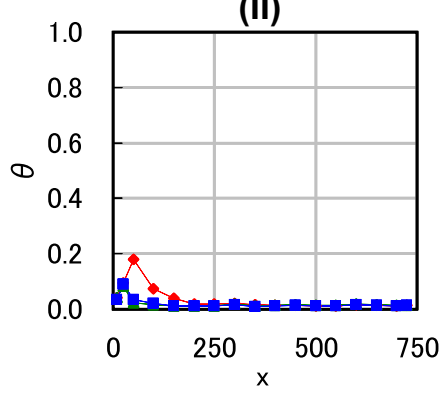

(i)

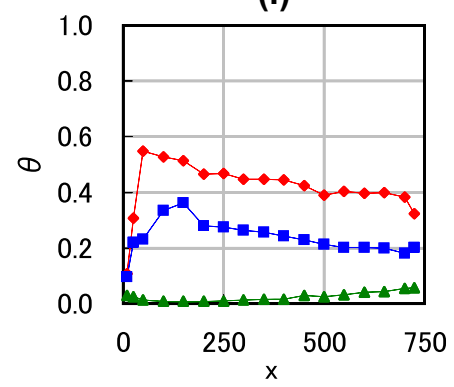

(ii)

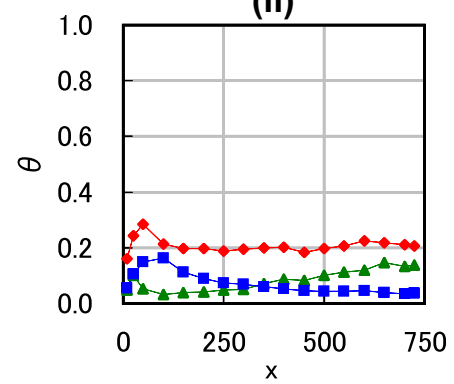

(iii)

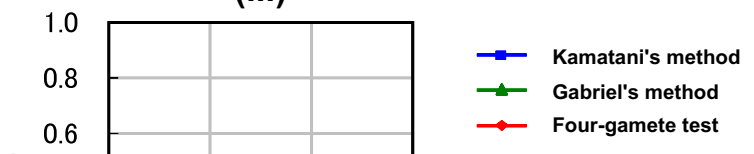

Figure 3

The effect of sample size on haplotype block characteristics under different block definitions: $\theta$ profiles versus sample number $(x)$. The number of repeat times $(n)$ was adjusted to $3,000(N=3,000)$. The labels of analyzed SNPs intervals are identical to those in Figure I. Sample size $(x)$ is shown on the horizontal axis. $\theta$ profiles (ratio of times to $N$ times when simulating the transition zone of haplotype block) are shown on the vertical axis. The three labels represent different haplotype block definitions: blue square, Kamatani's method; green triangle, Gabriel's method; red diamond, four-gamete test.

required to reach the plateau value for precise evaluation of haplotype block remains unclear.

$\theta$ profiles were also generated after changing the number of simulation times $(n)$ up to the maximum repeat times of 10,000 (Fig. 4). The $\theta$ value converged and reached the plateau value in all algorithms when simulations were repeated 2,500 times. Thus, the $\theta$ value with 3,000 repeat times was reliable for block identification with the plateau value in the simulation. In the simulation of 50 samples (dashed lines), the $\theta$ value was not equal to the result in 725 samples (solid lines), even if the repeat times increased to the maximum $(10,000)$. As a whole, these observations indicate that a sample size greater than 725 with a computational simulation of 3,000 times is required to obtain a converged $\theta$ value.

\section{Discussion}

Given the importance of haplotype block for genomewide association mapping, there is tremendous interest in constructing a haplotype block of the human genome. As a consequence, several questions have been raised about the nature of these blocks $[3,4,6]$. First, there are a number of variations in haplotype blocks based on the different block algorithms. In addition, several genetic factors, such as population, genetic marker density, and marker allele frequency, have contributed to the characteristics of haplotype blocks. However, it is not well known how many samples are sufficient to obtain reliable block characteristics $[5,9,11]$. To address this question, we genotyped 725 Japanese subjects with $>1,000$ SNPs on chromosome 20q. Additionally, we exploited a simple but refined simulation, which provided an original measurement ( $\theta$ value) generated by random re-sampling from the real genotyping data.

The simulated data were derived from a single population, hence our study does not address all ethnic groups. However, we found that the number of samples had an effect on the characteristics of haplotype block, even in a limited region of the human chromosome in a single population. Indeed, the $\theta$ value could not reach plateau with less than 200 samples in some parts of the analyzed region. Recent works also indicated that sample size has a marked effect on the detection of observed haplotype blocks $[5,11]$. That study showed that a large sample size was required to minimize the bias in means based on $\left|\mathrm{D}^{\prime}\right|$, while trying to reduce the bias by bootstrapping [12]. To identify more general properties of haplotype block, a relatively larger number of samples would be required than those in this 

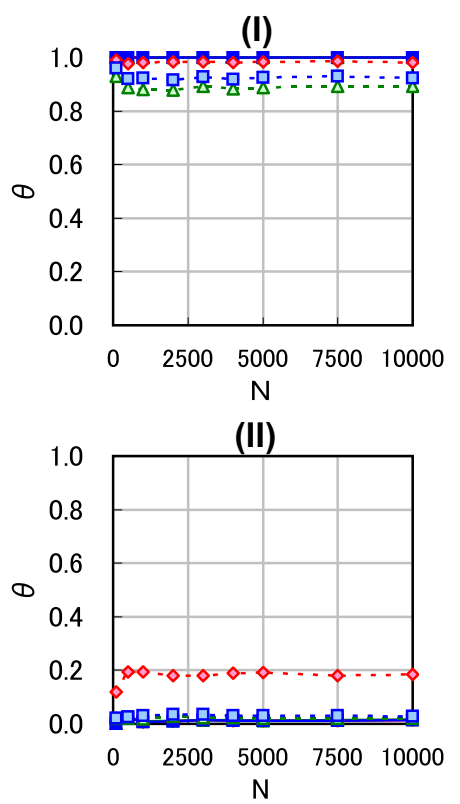

(i)

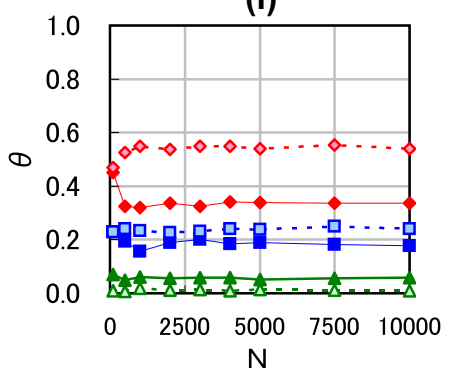

(ii)

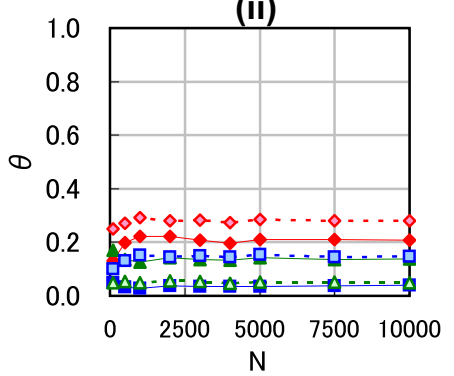

(iii)

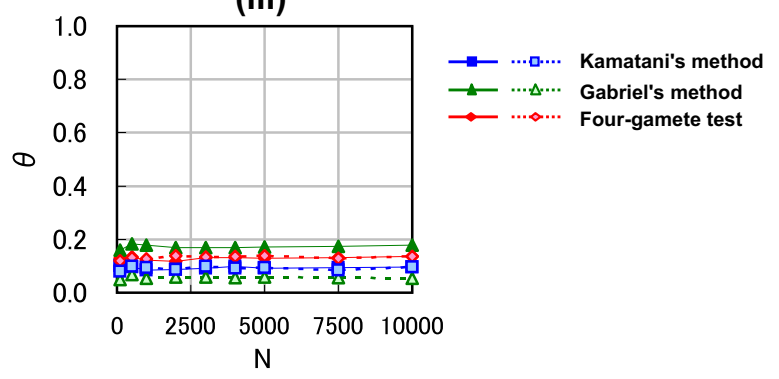

(iv)

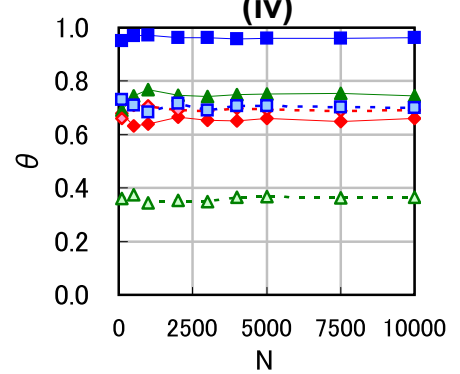

Figure 4

The effect of the number of simulation repeats on haplotype block characteristics under different block definitions: $\theta$ profiles versus the number of repeat times $(n)$. The sample size $(x)$ was adjusted by fixing it at 725 (solid lines) or 50 (dashed lines). The labels of analyzed SNPs intervals are identical to those in Figure I. The number of repeat times $(n)$ is shown on the horizontal axis. The simulation was repeated 10,000 times $(N=10,000)$ from I, I00, 500, I,000, 2,000, 3,000, 4,000, 5,000, and 7,500 times. $\theta$ profiles (the ratio of times to $N$ times when simulating the transition zone of haplotype block) are shown on the vertical axis. The three labels represent different haplotype block definitions: blue square, Kamatani's method; green triangle, Gabriel's method; red diamond, four-gamete test.

study. However, it is not feasible to increase the number of samples. Information on the variation of haplotype blocks is based on a trade-off between the accuracy of description, i.e., how much loss of information is acceptable, and the genotyping efforts needed to achieve this accuracy [5].

Another important and unsolved issue is the extent of block boundary conservation; namely, how fragile is the haplotype structure? To address this question, three different definitions of haplotype blocks were employed [13]. However, the primary concept of three algorithms was based on $\left|D^{\prime}\right|$ and focused on historical recombination, not allelic association. We could not avoid any bias resulting from a particular method. Although concordance of haplotype blocks exists in some regions, they are not common and might break up even if the region shows a single strong $\left|\mathrm{D}^{\prime}\right|$ value [14]. As described above, the block did not have absolute boundaries and might be defined in different ways. In addition, our simulation provides only one of many possible approaches. It is difficult to compare the advantage of these algorithms because block identification was influenced by thresholds in the algo- rithms. It remains unclear how best to merge or integrate block definitions from different algorithms. We will not discuss the advantage of a particular algorithm as we view this as a separate problem.

One potential weakness is a possible ascertainment bias against SNPs allele frequency, which could influence haplotype block characteristics. SNPs with minor allele frequency greater than $10 \%$ were selected in this analysis, underscoring the variety of haplotype blocks. Therefore, our results cannot be used to infer the complete nature of haplotype blocks, because we referred to limited haplotype diversity from a bias in common SNPs.

\section{Conclusion}

We developed a computational simulation that provides a realistic estimation of the observed genotyping data and attempted to unravel the underlying complexity of haplotype block. Based on this simulation, the sample size had an effect on the inferred haplotype block structure. For a valid description of haplotype blocks, further study will be required with a larger number of samples than presented here. 


\section{Methods}

\section{Preparation of genotyping data}

Genomic DNA was collected from 725 Japanese subjects (male/female; 336/389), consisting of 358 healthy control subjects (male/female; 145/213) and 367 type 2 diabetic patients (male/female; 191/176). An examination of birthplace information established that all subjects were of full Japanese ancestry. Detailed clinical information of samples was previously described (Additional file 2) [15]. A total of 1,147 SNPs were selected in a $19.31 \mathrm{Mb}$ region on chromosome 20q11.21-13.13 between D20S195 and D20S196. These SNPs were genotyped using a TaqMan SNP Genotyping Assay (Applied Biosystems, Foster City, CA) or a QuantiTect Probe PCR kit (Qiagen, Stanford, CA) according to the manufacturer's protocol. The fluorescence of VIC and FAM was measured with an ABI Prism 7900HT using Sequence Detector System (SDS) version 2.1 software (both from Applied Biosystems). The accuracy of genotyping was assessed by PCR-direct sequencing and showed $100 \%$ concordance as previously reported [15-17].

With the TaqMan method, genotype calls were identified by clustering the fluorescence intensity measurements for each SNP. All SNPs that were not members of genotyped clusters were eliminated. Expected genotyping accuracies were estimated with the quality score algorithm in SDS version 2.1. The intensity measurements were carefully checked by two independent researchers. In addition, the deviations of genotype distributions were evaluated by the Hardy-Weinberg equilibrium (HWE) test. This test was effective for identifying artifacts and improving data quality. Collectively, quality control was dependent on the reliability of the intensity measurements. The criteria used to judge reliability included calling rate, number of genotyped clusters, and consistency with the HWE test. Of the 1,147 SNPs genotyped, 103 were excluded and 1,044 passed the quality control criteria. An integrated system of Fujitsu Gene Discovery System (FGDS) version 2.0 (Fujitsu Ltd, Tokyo, Japan) was developed to deliver highquality genotyping data [15-17].

\section{Linkage disequilibrium (LD) on chromosome 20q I I.2 I- 13.13}

Linkage disequilibrium (LD) features along a $19.31 \mathrm{Mb}$ contiguous segment on chromosome 20q11.21-13.13 were systematically characterized. FGDS version 2.0 was used to calculate the pairwise LD coefficients, $\left|D^{\prime}\right|$ and $r^{2}$ for the 1,044 SNPs that passed the quality control (Additional file 3 ).

The pairwise LD coefficient is the difference in haplotype frequency between the estimation of the expectation-maximization (EM) algorithm and calculation of the multiplication of allele frequencies. This coefficient is given as

$$
\mathrm{D}=\mathrm{x} 11-\mathrm{p} 1 \mathrm{q} 1,
$$

where $\mathrm{p} 1$ and $\mathrm{q} 1$ are the frequencies of alleles $\mathrm{A} 1$ and $\mathrm{B} 1$ at loci $\mathrm{A}$ and $\mathrm{B}$, and $\mathrm{x} 11$ is the frequency of haplotype A1B1. The standardized LD coefficient, $\mathrm{r}^{2}$, was calculated by the following formula [18]:

$$
\mathrm{r}^{2}=\mathrm{D}^{2} /(\mathrm{p} 1 \mathrm{p} 2 \mathrm{q} 1 \mathrm{q} 2)
$$

where $\mathrm{p} 2$ and $\mathrm{q} 2$ are the frequencies of the other alleles at loci A and B. Lewontin's coefficient, |D'|, is described by the following formula:

$$
\left|D^{\prime}\right|=\operatorname{abs}\left(D^{\prime}\right), D^{\prime}=D / D_{\text {max }}
$$

where $\mathrm{D}_{\max }=$ minimum $(\mathrm{p} 1 \mathrm{q} 2, \mathrm{p} 2 \mathrm{q} 1)$ when $\mathrm{D}$ is $<0$, and where $D_{\max }=\operatorname{minimum}(\mathrm{p} 1 \mathrm{q} 1, \mathrm{p} 2 \mathrm{q} 2)$ when $\mathrm{D}$ is $>0$ [19].

\section{Computational simulation}

Figure 2 is a flow chart showing the procedure of computational simulation. The simulation consisted of re-sampling, haplotype block definition, and block concordance, including the process evaluating the effect of sample size $(x)$.

\section{Re-sampling}

A re-sampling method was developed to replace the real genotyping data for assessment of the variation of haplotype blocks based on sample size. Sample size was ran-

Table 2: Thresholds of three different algorithms to infer haplotype block

\begin{tabular}{lll}
\hline Algorithms & Thresholds \\
\hline I Four-gametes test (recombination-based) & Threshold of forth gamete's haplotype frequency: 0.01 \\
2 Gabriel's method (LD-based) & Confidence interval threshold for strong LD (lower side): 0.70 \\
& Confidence interval threshold for strong LD (upper side): 0.98 \\
& Upper confidence interval maximum for strong recombination: 0.90 \\
& Threshold of strong LD rate in region: 0.95
\end{tabular}


domly selected on the basis of SNP markers to permit the same sampling from the genotyping data from the 725 original samples. This re-sampling method is quite similar to the Mersenne Twister bootstrap method with random sampling for the generation of random numbers [20].

The conditions of HWE could change in the simulation with re-sampling, although the consistency of HWE existed in the genotyping data from the original 725 samples. SNPs with Chi-square $\mathrm{P}<0.05$ by HWE test were excluded from the simulation data to reduce the influence of HWE. Similarly, SNPs with minor allele frequencies (MAF) less than 0.10 were excluded from the simulation data.

\section{Haplotype block definition}

One purpose of the simulation was to evaluate the transition zone of the haplotype block. The transition zone of $\mathrm{LD}$, in which continuous high LD values were disrupted by low LD values, was examined first [21].

Three different algorithms, recombination-based [9], LDbased [4], and diversity-based [22], were employed to define the haplotype block. The thresholds of three algorithms are shown in Table 2. Each algorithm provided different and complementary concepts. These three algorithms are described in detail in original papers and are briefly summarized below.

For the recombination-based method, a four-gamete test identifies a haplotype block as having the lowest frequency among the four phases of haplotypes consisting of pairwise SNPs [9]. The generation of genetic recombination in an interval of pairwise SNPs was indicated when the lowest frequency was greater than a threshold. Recombination was considered to have occurred if there were four haplotypes for any marker pair.

The LD-based method, Gabriel's method, is based on the LD coefficient $\left|D^{\prime}\right|$ and the $95 \%$ confidence interval (CI) [4]. Pairwise SNPs have a strong LD when the upper-side of $95 \% \mathrm{CI}$ of $\left|\mathrm{D}^{\prime}\right|$ is over 0.98 , and the lower-side is over 0.70. In contrast, strong historical recombination exists when the upper-side of $95 \% \mathrm{CI}$ of $\left|\mathrm{D}^{\prime}\right|$ is less than 0.90. A haplotype block is defined as a region in which a small proportion (under 0.05) of SNPs shows strong historical recombination.

The foundations of the diversity-based Kamatani's method are the LD coefficient $\left|\mathrm{D}^{\prime}\right|$ and haplotype frequency [22]. The haplotype block was constructed in two steps. First, an initial haplotype block was made consisting of all pairwise SNPs, in which $\left|\mathrm{D}^{\prime}\right|$ was over 0.90 . Using all SNPs in the initial block, the major haplotypes are identified by estimating the phase and frequency of haplotypes. In the second step, an adjacent SNP is added to the initial haplotype block, and the phase and frequency of haplotypes are estimated again. If another major haplotype is not recognized, an adjacent SNP is included in the initial haplotype block. The second step is repeated in the $5^{\prime}$ and $3^{\prime}$ directions until an additional major haplotype is generated. SNPHAP version 1.3.1 [23] was used to estimate haplotype phase and frequency.

\section{Haplotype block concordance}

The simulation procedure was repeated $N$ times, with $N$ ranging from 1 to 10,000 . The incremental parameter of sampling times is represented as $n$. Original measurement for the identification of haplotype block, $\theta$ and $\rho$, were introduced to measure the correlation in haplotype structure between pairwise SNPs and provide a degree of confidence.

Let $\mathrm{SNP}_{j, k}$ denote the interval between $\mathrm{SNP}_{j}$ and $\mathrm{SNP}_{k}$. The number of times that each $\mathrm{SNP}_{j, k}$ interval was included in or excluded from a reference haplotype block was calculated across all re-sampling samples. The reference haplotype block was defined using all 725 samples with each algorithm (Fig. 1B). The value obtained when $\mathrm{SNP}_{j, k}$ was included in a transition zone of block (i. e., excluded from a reference block) was defined as $\theta_{j, k^{\prime}}$ the ratio of times to the total number of $N$ times of the simulation. The relationship between $\theta_{j, k}$ and $\rho_{j, k}$ was as follows:

$$
\theta_{j, k}=\sum_{n=1}^{N} \rho_{j, k}^{n} / N .
$$

Let $\rho_{j, k}$, which shows a value of 1 or 0 , denote $\mathrm{SNP}_{j, k}$ that is or is not included in the transition zone. When $\rho_{j, k}=1$, $\mathrm{SNP}_{j, k}$ is within the transition zone (i.e., excluded from the reference haplotype block defined by 725 samples). When $\rho_{j, k}=0, \mathrm{SNP}_{j, k}$ is outside the transition zone (i.e., included in the reference haplotype block defined by 725 samples). Let $\rho_{j, k}^{n}$ denote the $n$th data of $\rho_{j, k}$ in the total re-sampling number of $N$ times. All profiles of $\theta$ were plotted as $\theta_{j, k}$ against the physical position of SNPs sliding from $j=$ A001 and $k=\mathrm{A} 002$ to $j=\mathrm{A} 036$ and $k=\mathrm{A} 037$.

In this analysis, all algorithms were implemented in Linux OS (Red Hat 9) using Perl. The simulation was run on an IA server with four $3.20 \mathrm{GHz}$ Intel (R) Pentium (R) processors and $500 \mathrm{MB}$ of RAM.

\section{Authors' contributions}

DO developed the computational simulation method, and TT genotyped all DNA samples. DO and TT performed all statistical analyses. TT, DO and MI drafted the 
manuscript. NN, TY and HS cooperated the collection of DNA samples. All authors approved the final manuscript.

\section{Additional material}

\section{Additional file 1}

Supplementary Table 1 shows the mean square errors (MSE) in estimating the effect of the mixture of cases with controls.

Click here for file

[http://www.biomedcentral.com/content/supplementary/1471-

2105-8-200-S1.pdf]

\section{Additional file 2}

Supplementary Table 2 shows the summary of 725 Japanese samples. Click here for file

[http://www.biomedcentral.com/content/supplementary/14712105-8-200-S2.pdf]

\section{Additional file 3}

Supplementary Figure 1 shows the entire plot of linkage disequilibrium (LD) coefficient $\left|D^{\prime}\right|$ across chromosome 20q11.21-13.13 (19.31 Mb). Click here for file

[http://www.biomedcentral.com/content/supplementary/14712105-8-200-S3.pdf]

\section{Acknowledgements}

This study was supported by grants from the Japan Biological Information Consortium affiliated with the New Energy and Industrial Technology Development Organization, Japan Society for the Promotion of Science (Grant for Genome Research of the Research for the Future Program), and Cooperative Link of Unique Science and Technology for Economy Revitalization (CLUSTER, Tokushima, Japan).

\section{References}

I. Hirschhorn JN, Daly MJ: Genome-wide association studies for common diseases and complex traits. Nat Rev Genet 2005, 6(2):95- 108

2. Patil N, Berno AJ, Hinds DA, Barrett WA, Doshi JM, Hacker CR, Kautzer CR, Lee DH, Marjoribanks C, McDonough DP, Nguyen BT, Norris MC, Sheehan JB, Shen N, Stern D, Stokowski RP, Thomas DJ, Trulson MO, Vyas KR, Frazer KA, Fodor SP, Cox DR: Blocks of limited haplotype diversity revealed by high-resolution scanning of human chromosome 2I. Science 200I, 294(5547): I7I9-23.

3. Daly MJ, Rioux JD, Schaffner SF, Hudson TJ, Lander ES: High-resolution haplotype structure in the human genome. Nat Genet 200I, 29(2):229-32.

4. Gabriel SB, Schaffner SF, Nguyen H, Moore JM, Roy J, Blumenstiel B, Higgins J, DeFelice M, Lochner A, Faggart M, Liu-Cordero SN, Rotimi C, Adeyemo A, Cooper R, Ward R, Lander ES, Daly MJ, Altshuler D: The structure of haplotype blocks in the human genome. Science 2002, 296:2225-29.

5. Nothnagel M, Rohde K: The effect of single-nucleotide polymorphism marker selection on patterns of haplotype blocks and haplotype frequency estimates. Am J Hum Genet 2005, 77:988-98.

6. International HapMap Consortium: A Haplotype map of the human genome. Nature 2005, 27:1299-1320.

7. Phillips MS, Lawrence R, Sachidanandam R, Morris AP, Balding DJ, Donaldson MA, Studebaker JF, Ankener WM, Alfisi SV, Kuo FS, Camisa AL, Pazorov V, Scott KE, Carey BJ, Faith J, Katari G, Bhatti HA, Cyr JM, Derohannessian V, Elosua C, Forman AM, Grecco NM, Hock CR, Kuebler JM, Lathrop JA, Mockler MA, Nachtman EP, Restine SL, Varde SA, Hozza MJ, Gelfand CA, Broxholme J, Abecasis GR, Boyce-Jacino MT, Cardon LR: Chromosome-wide distribution of haplotype blocks and the role of recombination hot spots. Nat Genet 2003, 33(3):382-87.

8. Ke X, Hunt S, Tapper W, Lawrence R, Stavrides G, Ghori J, Whittaker P, Collins A, Morris AP, Bentley D, Cardon LR, Deloukas P: The impact of SNP density on fine-scale patterns of linkage disequilibrium. Hum Mol Genet 2004, 13:577-88.

9. Wang N, Akey JM, Zhang K, Chakraborty R, Jin L: Distribution of recombination crossovers and the origin of haplotype blocks: the interplay of population history, recombination, and mutation. Am J Hum Genet 2002, 7 I: 1227-34.

10. Fallin D, Schork NJ: Accuracy of haplotype frequency estimation for biallelic loci, via the expectation-maximization algorithm for unphased diploid genotype data. Am J Hum Genet 2000, 67(4):947-59.

II. Sun X, Stephens JC, Zhao H: The impact of sample size and marker selection on the study of haplotype structures. Hum Genomics 2004, I(3): 179-193.

12. Teare MD, Dunning AM, Durocher F, Rennart G, Easton DF: Sampling distribution of summary linkage disequilibrium measures. Ann Hum Genet 2002, 66(Pt 3):223-33.

13. Indap AR, Marth GT, Struble CA, Tonellato P, Olivier M: Analysis of concordance of different haplotype block partitioning algorithms. BMC Bioinformatics 6:303. 2005, Dec 15

14. Ding K, Zhou K, Zhang J, Knight J, Zhang X, Shen Y: The effect of haplotype-block definitions on inference of haplotype-block structure and htSNPs selection. Mol Biol Evol 2005, 22(1): 148-59.

15. Tanahashi T, Osabe D, Nomura K, Shinohara S, Kato H, Ichiishi E, Nakamura N, Yoshikawa T, Takata Y, Miyamoto T, Shiota H, Keshavarz P, Yamaguchi Y, Kunika K, Moritani M, Inoue H, Itakura M: Association study on chromosome $20 \mathrm{qI} / .2 \mathrm{I}-13.13$ locus and its contribution to type 2 diabetes susceptibility in Japanese. Hum Genet 2006, I 20(4):527-42.

16. Kato H, Nomura K, Osabe D, Shinohara S, Mizumori O, Katashima R, Iwasaki S, Nishimura K, Yoshino M, Kobori M, Ichiishi E, Nakamura N, Yoshikawa T, Tanahashi T, Keshavarz P, Kunika K, Moritani M, Kudo E, Tsugawa K, Takata Y, Hamada D, Yasui N, Miyamoto T, Shiota $\mathrm{H}$, Inoue $\mathrm{H}$, Itakura M: Association of single-nucleotide polymorphisms in the suppressor of cytokine signaling 2 (SOCS2) gene with type 2 diabetes in the Japanese. Genomics 2006, 87:446-58.

17. Hamada D, Takata $Y$, Osabe D, Nomura K, Shinohara S, Egawa H, Nakano S, Shinomiya F, Scafe CR, Reeve VM, Miyamoto T, Moritani M, Kunika K, Inoue H, Yasui N, Itakura M: Association between single-nucleotide polymorphisms in the SEC8LI gene, which encodes a subunit of the exocyst complex, and rheumatoid arthritis in a Japanese population. Arthritis Rheum 2005, 52:137|-80.

18. Hill WG, Robertson A: Linkage disequilibrium in finite populations. Theor Appl Genet 1968, 38:226-31.

19. Lewontin RC: The interaction of selection and linkage. I. General considerations: heterotic models. Genetics 1964, 49:49-67.

20. Matsumoto M, Nishimura T: Mersenne Twister: A 623-dimensionally equidistributed uniform pseudorandom number generator. ACM Trans on Modeling and Computer Simulation 1998, 8:3-30.

21. Schwartz R, Halldorsson BV, Bafna V, Clark AG, Istrail S: Robustness of inference of haplotype block structure. J Comput Biol 2003, IO(I):13-19.

22. Kamatani N, Sekine A, Kitamoto T, lida A, Saito S, Kogame A, Inoue $E$, Kawamoto $M$, Harigai $M$, Nakamura $Y$ : Large-scale singlenucleotide polymorphism (SNP) and haplotype analyses, using dense SNP Maps, of 199 drug-related genes in 752 subjects: the analysis of the association between uncommon SNPs within haplotype blocks and the haplotypes constructed with haplotype-tagging SNPs. Am J Hum Genet 2004, 75:190-203.

23. SNPHAP version 1.3.I [http://www-gene.cimr.cam.ac.uk/clayton/ software/] 